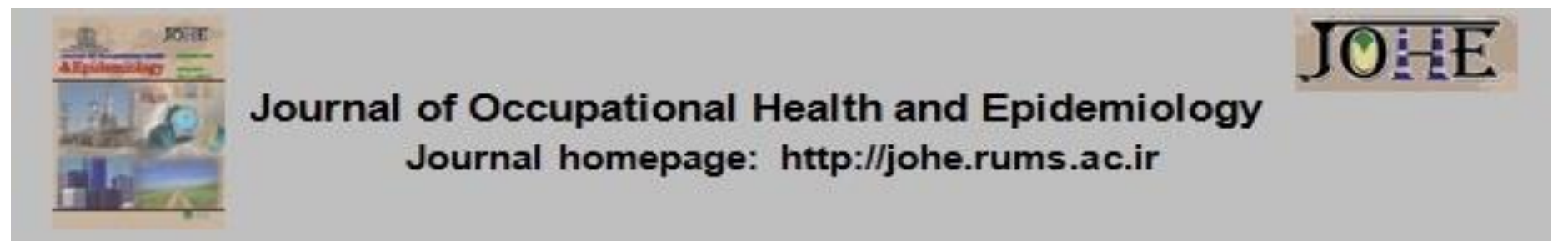

\title{
The Validity and Reliability of the Persian Version of the Impact on Participation and Autonomy (IPA-p) Scale in Patients with Positive Angiography
}

\author{
Zohreh Mordouei ${ }^{1}$, Mahmood Sheikhfathollahi², Mohsen Rezaeian ${ }^{3}$, Hasan Ahmadinia ${ }^{4}$, Mohsen \\ Mirzaei $^{5}$, Mohammad Safarian ${ }^{6}$, Reza Vazirinejad ${ }^{7 *}$ \\ 1. MSc in Epidemiology, School of Medicine, Rafsanjan University of Medical Sciences, Rafsanjan, Iran. \\ 2. Assistant Prof., Dept. of Epidemiology and Biostatistics, School of Medicine, Rafsanjan University of Medical Sciences, Rafsanjan, \\ Iran. \\ 3. Professor, Dept. of Epidemiology and Biostatistics, Occupational Environmental Research Center, School of Medicine, Rafsanjan \\ University of Medical Sciences, Rafsanjan, Iran. \\ 4. Assistant Prof., Dept. of Epidemiology and Biostatistics, Occupational Environmental Research Center, School of Medicine, \\ Rafsanjan University of Medical Sciences, Rafsanjan, Iran. \\ 5. Assistant Prof., Dept. of Cardiology Medicine, Medical School, Rafsanjan University of Medical Sciences, Rafsanjan, Iran. \\ 6. Assistant Prof., Dept. of Cardiology Medicine, Medical School, Rafsanjan University of Medical Sciences, Rafsanjan, Iran. \\ 7. Professor, Dept. of Social Medicine, Social Determinants of Health Research Center, Rafsanjan University of Medical Sciences, \\ Rafsanjan, Iran.
}

Citation: Mordouei Z, Sheikhfathollahi M, Rezaeian M, Ahmadinia H, Mirzaei M, Safarian M,
Vazirinejad R. The Validity and Reliability of the Persian Version of the Impact on
Participation and Autonomy (IPA-p) Scale in Patients with Positive Angiography. J Occu
Health Epidemiol 2021; 10(4):217-23.

\section{Article Info}

* Corresponding author:

Reza Vazirinejad,

E-mail:

rvazirinejad@yahoo.co.uk

\begin{abstract}
Article history
Received: May 2021

Accepted: Dec 2021
\end{abstract}

$10.52547 /$ johe.10.4.217

Print ISSN: 2251-8096 Online ISSN: 2252-0902

Peer review under responsibility of Journal of Occupational Health and Epidemiology

\section{Abstract}

Background: Cardiovascular disease often leads to limitations in patient activities. Therefore, providing a valid and reliable tool to measure the level of participation and autonomy can be used for improving their health. This study aimed to determine the validity and reliability of the Persian version of a participation and autonomy scale (IPA-p) in patients with positive angiography.

Materials and Methods: This psychometric properties assessment study was conducted recruiting 202 positive angiography patients referred to a cardiovascular diseases clinic in Rafsanjan in 2017. Ten experts were also recruited to help assess the face and content validity. Criterion validity of the scale was assessed by calculating the correlation of the two sets of scores collected from patients and their relatives. To compute the construct validity, exploratory and confirmatory factor analysis were performed. Scale reliability was also evaluated.

Results: The content validity index was 0.94 . The correlation coefficient between the scores of the completed questionnaires by patients and their relatives ranged between 0.443 and 1.0. Exploratory factor analysis extracted 9 factors, explaining about $73 \%$ of the total variance of the scale. Confirmatory factor analysis confirmed the fitting of the model. The Cronbach's alpha coefficient was 0.962, the coefficient of Split-half was 0.825 , the Intera-class correlation coefficient was 0.363 , and the coefficient of Test-retest was 0.807 .

Conclusion: Present findings the Persian version of the IPA scale to be a valid and reliable questionnaire for assessing participation and autonomy in patients with positive angiography. More investigation is recommended.

Keywords: Cardiovascular Disease, Validity, Reliability, Factor Analysis.

\section{Introduction}

Cardiac attacks usually occur acutely, with the obstruction of blood vessels being their main cause [1]. Several environmental and genetic factors are responsible for the occurrence of cardiovascular disease (CVD) [2]. The most 
important complication of CVD is congestive heart failure, leading to disability and hospitalization of patients [3]. Thus, a large number of patients spend a long time of their life with both physical and psychological limitations due to the disease.

Concepts such as disability [4], impairment $[5,6]$, handicap [6], and quality of life (QOL) [7] have been widely used to assess the impact of ailments and injuries, including cardiovascular disease, on human health. Some of these concepts are criticized for their negative impression, and QOL is weak because of its subjective assessment. A suitable scale should have a positive effect and also be able to assess human health objectively [8].

Regarding these limitations, a new generation of health assessment scales was introduced about two decades ago with a core concept of Participation and Autonomy. One of the first questionnaires for measuring the impact of disease on patients' health was designed by Cardol et al., namely the Impact on Participation and Autonomy (IPA) Scale. This questionnaire was intended to evaluate the participation and autonomy of Multiple Sclerosis patients objectively [9]. It is supposed to help scientists measure the level of health of patients in terms of their ability to live in different situations. In other words, the disease imposes some limitations on the individuals by which, in return, it would be impossible to fulfill their roles in their life (in all/some parts). Each particular disease (such as CVD) would affect special aspects of patients' life. In order to improve patients' health, it would be helpful if clinicians detect affected situations that are more limited. A suitable scale could help service providers distinguish the real aspects of patients' life needing improvement. Since CVD as a group of common chronic diseases causes some limitations in patients' life, a proper scale to measure the level of its disablement in different aspects can help cardiologists and other service providers to improve patients' health. An effective scale supposed to be helpful should have many positive characteristics. Not only should it have acceptable psychometric properties (e.g., validity and reliability), but it also should be well designed based on the culture, literature, and some other characteristic of the respondents [10].

Since an effective assessment tool in the form of a questionnaire must be valid, reliable, and unbiased, this study aims to introduce and prepare a valid and reliable questionnaire to measure the level of participation and autonomy of Persian patients with cardiovascular disease. This scale is supposed to help cardiologists and other stakeholders provide appropriate treatment and sufficient health services to improve the health of CVD patients. This study intends to determine the validity and reliability of the Persian version of a participation and autonomy scale (IPA-p) in patients with positive angiography.

\section{Materials and Methods}

This psychometric properties assessment study was conducted to determine the validity (face, content, construct, and criterion) and reliability of the Persian version of the IPA questionnaire in patients with positive coronary angiography in Rafsanjan in 2017. The Ethics Approval No was IR. RUMS. REC.1396.49.

There were two groups of respondents in our study: a) ten experts with different disciplines were invited for assessing face validity, content validity ratio (CVR), and content validity index (CVI), b) two hundred and two people with positive coronary angiography results and their relatives were recruited for measuring construct and criterion validity and reliability.

According to the sample size determination method in the factor analysis, the minimum required sample size is 5 to 10 people for each subscale in the questionnaire [11]. The questionnaire had 9 original parts and 1 section added to the Persian version of the scale [12]. Thus, the minimum number of respondents required in this study was 100 . Regarding a response rate of $50 \%, 202$ patients were invited and requested to sign the consent form. Respondents were randomly selected from the list of patients with positive coronary angiography in Ali Ebne Abitalib Hospital. The inclusion criteria included positive coronary angiography confirmed by a cardiologist and age over 18 . The exclusion criteria included age less than 18 years and dissatisfaction with participation in this study.

Since the cultural background of Persian respondents was different from those whom the original IPA scale was designed for and some of the questions seemed to be unfamiliar to patients, this questionnaire was literally localized for the present target population after its forward and backward translation. For instance, some words of the original IPA were replaced with completely different words with the same concept among Persian CVD patients. At last, the final version of the questionnaire (IPA-p) was prepared after accurate literary editing.

Ten experts in four fields of psychology, biostatistics, epidemiology, and cardiology were invited to evaluate the face validity. The experts completed a face validity evaluation checklist containing items about the simplicity of questions, 
comprehensibility of questions, and the formulation of items.

The content validity ratio (CVR) and content validity index $(\mathrm{CVI})$ were both calculated using their view. After careful review of IPA-p by the experts, the relevance of each item to the theoretical domains and its appropriateness, the necessity of each item was scored as "1- not necessary, 2- not necessary but useful, and 3necessary". Based on these scores, CVR for each item was calculated using the following formula: (where "ne" was the number of experts who gave a score of 3 to the item (e.g., the "necessary" choice), and $\mathrm{N}$ was the total number of experts) [13].

Formula 1.

$$
C V R=\frac{n e-\frac{N}{2}}{\frac{N}{2}}
$$

Then, CVI for all items was calculated (e.g., the average of CVRs) [14]. In content validity assessment, according to the Lawshe table, based on the evaluation of 11 experts, a CVR higher than 0.59 and a minimum $\mathrm{CVI}$ value of 0.70 is required [13].

To assess construct validity, both materials and factor analysis were conducted. In the former, the correlation between the score of each item and the total score of the related domain, as well as the correlation between the mean scores of each domain with the total score of the scale, were calculated [15]. Exploratory factor analysis was performed to confirm the factor structure of the scale. In order to check the adequacy of the sample size, index Kaiser-Meyer-Olkin measure (KMO) was used; further, to assess the significance of the matrix correlation data, Bartlett's test of sphericity was used. The latter was performed using Principal Components and Varimax rotation. The factor extraction criterion, the scree plot, and the Eigenvalues of more than one were considered the main factors [16]. Confirmatory factor analysis was then used to examine the fitting of the scale and confirm the results of the exploratory factor analysis.

Fitness indicators fall into three groups: Absolute, Comparative, and Parsimonious, each of which provides different information about the fitness and appropriateness of the model. In this analysis, a) the chi-square/degree of freedom (CMIN / DF) is less than $3, b)$ the Tucker-Lewis index (TLI), the normed fit index (NFI), Comparative fit index (CFI), and Incremental fit index (IFI) are greater than
0.90, c) Parsimonious normed fit index (PNFI) and adaptive fit index (Parsimonious comparative fit index; PCFI) are greater than 0.50, and d) the root mean square error of approximation (RMSEA) is less than 0.08 .

In order to evaluate the Construct and Criterion validity of the IPA-p scale, patients $(n=202)$ were asked to complete the questionnaire. Patients who were not literate enough to read the questions were assisted by patients' companions or study interviewer. In the absence of a golden test needed for assessing the scale criterion validity, the research team decided to use the objective data collected from patient's relatives. Thus, the relatives who were living with patients (wife, husband, son and daughter) were requested to complete a copy of IPA-p for answering the items about patients' performance in different domains. The correlation between the scores of the different domains in the questionnaires completed by patients and their relatives was used to measure the criterion validity of IPA- $p$ [17].

To evaluate the reliability of IPA- $p$, the scale was again completed by the same 202 participants in 30-45 days after the first scale completion. Respondents who did not refer to the clinic at the time of the second completion were contacted through telephone calls. After completing IPA-p on the second occasion, the Intera-class correlation coefficient (ICC), Split-half, Test-retest, and Cronbach's alpha coefficient were computed to measure the reliability of the scale. Information about some demographic factors, including age, gender, educational level, occupation, marital status, economic status, and family history of heart disease, were also obtained.

Statistical analysis was performed using SPSS version 21 and Amos version 20. Also, the significance level was 0.05 .

\section{Results}

Mean age of patients was $61.14 \pm 11.24$ years ( $\min =29$ and $\max =91$ years). Of the patients, $122(60.4 \%)$ were male, 200 (99\%) married, 94 $(46.5 \%)$ illiterate, and also $64(31.7 \%)$ had no degree, 32 (15.8\%) had diploma, 5 (2.5\%) associate's degree, $4(0.23 \%$ a bachelor's degree, and $3(1.5 \%)$ a master's degree. Twenty-eight people $(13.9 \%)$ were unemployed, 74 people $(36.6 \%)$ were housewives, 19 people $(9.4 \%)$ were employees, and 81 people (40.1\%) did not report having any specific job. About $50 \%$ of patients (99 people) reported a positive family history of heart disease.

To evaluate face validity, $80.0 \%(n=8)$ of experts reported the overall ability of IPA-p to assess the 
extent of participation and autonomy of CVD patients in their private and social life as "high and very high" Ninety percent $(n=9)$ of experts expressed the clarity and simplicity of IPA-p items to be "high and very high" Sixty percent $(n=6)$ of experts believed that the quality of sentence structure and comprehensibility of items were "high and very high" However, only $40.0 \%$ of experts (n $=4$ ) announced that the number of IPA-p items was sufficient. The ability of the questionnaire to evaluate its different domains based on experts' points of view is presented in Table 1.

Table 1. Frequency distribution of experts based on their points of view about the ability of the IPA-p items to evaluate its domains among respondents $(n=202)$

\begin{tabular}{|c|c|c|c|c|c|}
\hline Domains & $\begin{array}{c}\text { Very low } \\
\text { n (\%) }\end{array}$ & $\begin{array}{l}\text { Low } \\
\text { n (\%) }\end{array}$ & $\begin{array}{c}\text { Moderate } \\
\text { n (\%) }\end{array}$ & $\begin{array}{l}\text { High } \\
\text { n (\%) }\end{array}$ & $\begin{array}{c}\text { Very high } \\
\text { n (\%) }\end{array}$ \\
\hline Mobility & $0(0)$ & $0(0)$ & $2(20)$ & $6(60)$ & $2(20)$ \\
\hline Self-care & $0(0)$ & $0(0)$ & $2(20)$ & $7(70)$ & $1(10)$ \\
\hline Household tasks and family role & $0(0)$ & $0(0)$ & $2(20)$ & $5(50)$ & $3(30)$ \\
\hline Looking after money & $0(0)$ & $1(10)$ & $5(50)$ & $2(20)$ & $2(20)$ \\
\hline $\begin{array}{c}\text { Leisure } \\
\end{array}$ & $0(0)$ & $0(0)$ & $5(50)$ & $3(30)$ & $2(20)$ \\
\hline Social relations & $0(0)$ & $1(10)$ & $0(0)$ & $6(60)$ & $3(30)$ \\
\hline $\begin{array}{l}\text { Paid work } \\
\end{array}$ & $0(0)$ & $0(0)$ & $5(50)$ & $4(40)$ & $1(10)$ \\
\hline Help and support of others & $0(0)$ & $2(20)$ & $4(40)$ & $3(30)$ & $1(10)$ \\
\hline Education and learning & $0(0)$ & $1(10)$ & $3(30)$ & $4(40)$ & $2(20)$ \\
\hline Religious affairs & $0(0)$ & $1(10)$ & $0(0)$ & $6(60)$ & $3(30)$ \\
\hline
\end{tabular}

Based on experts' points of view, CVR (for each item) and CVI (for the scale) were calculated for the IPA-p scale to measure its content validity. The results showed that the amounts of CVR for IPA-p items ranged from 0.63 to 1 . The amount of $\mathrm{CVI}$ was 0.94 , confirming the content validity of the IPA-p scale and the amounts of CVR.

Items of IPA-p belonged to 9 domains of "mobility", "self-care", "household tasks and family role", "looking after money", "social relations", "paid work", "religious affairs", and two new factors were extracted according to the exploratory factor analysis in the study (material, spiritual, educational needs, and the impact of the disease on various aspects of life). The results interestingly showed about $73 \%$ of the variance of the scale scores to be explained by these nine factors. The acceptable load factor was more than 0.4 [18].

In other words, the data collected by the IPA-p scale showed that all its items belonged to the related domains, confirming its construct validity. Further, the KMO value for the scale was 0.906 , indicating the adequacy of the sample size. In addition, the Bartlett value was 4668.015, which was statistically significant $(P<0.001)$. The results showed that the implementation of factor analysis for the obtained data could be justified [19].

The results of fitting goodness indexes based on the 9 factors model are presented in Table 2.

Table 2. General indexes of fitting the IPA-p questionnaire in patients with positive angiography $(n=202)$

\begin{tabular}{ccccccccc}
\hline Index & CMIN/ DF & TLI & IFI & NFI & CFI & PCFI & PNFI & RMSEA \\
Value & & & & & & & & \\
\hline Calculated & 1.484 & 0.924 & 0.936 & 0.828 & 0.935 & 0.802 & 0.710 & 0.049 \\
acceptable value & $<3$ & $>0.90$ & $>0.90$ & $>0.90$ & $>0.90$ & $>0.50$ & $>0.50$ & $<0.08$ \\
\hline
\end{tabular}

* CMIN/ DF: Chi-square/degree of freedom, TLI: Tucker-Lewis index, IFI: Incremental fit index, NFI: Normed fit index, CFI: Comparative fit index, PCFI: Parsimonious comparative fit index, PNFI: Parsimonious normed fit index, RMSEA: Root mean square error of approximation

According to the table, the model had a very good overall fit. In order to measure the construct validity of IPA-p, material analysis was conducted, and the correlation of the mean score of each domain with the total mean score of the scale was examined. The correlation coefficients ranged between 0.445 to 0.846 . These coefficients were all positive and statistically significant $(P<0.001)$ (Table 3$)$.
Given the need to assess criterion validity, the correlation coefficients of the mean scores of the ten domains obtained from patients with the mean scores of those obtained from patients' relatives $(n=202)$ were calculated. These correlation coefficients ranged between 0.443 and 1.0, indicating a moderate to perfect correlation (Table 3). 
Table 3. Correlation coefficients between mean scores of each domain obtained from patients and their relatives (criterion v.) and between the mean scores of each domain with the mean total score of the scale (construct v.) ( $n=202$ )

\begin{tabular}{ccc}
\hline Domains & Criterion validity & Construct validity \\
\hline Mobility & 0.711 & 0.809 \\
\hline Self-care & 0.755 & 0.819 \\
\hline Household tasks and family role & 0.764 & 0.846 \\
\hline Looking after money & 0.595 & 0.635 \\
\hline Leisure & 0.720 & 0.789 \\
\hline Social relations & 0.605 & 0.765 \\
\hline Paid work & 1 & 0.524 \\
\hline Help and support of others & 0.503 & 0.479 \\
\hline Education and learning & 0.443 & 0.445 \\
\hline Religious affairs & 0.663 & 0.734
\end{tabular}

The results indicated that Cronbach's alpha coefficient for the total scale was 0.962 . Split-half coefficients for domains ranged between 0.173 and 0.820 , and for the total scale was 0.825 . The amount of ICC was also 0.363 . The range of Testretest coefficients for ten domains was 0.270 to 0.736 , and for the total scale was 0.807 . The results confirmed IPA-p as a reliable scale for use among positive coronary angiography patients.

\section{Discussion}

Since no scale is introduced to measure the degree of participation and autonomy in patients with positive coronary angiography (as well as no other CVDs) on publication so far, this is the first study designed to provide a valid and reliable scale for this purpose. The initial stage of our research showed that IPA-p had been designed properly and its items had been well situated. Further, $80 \%$ of experts in our study believed that the IPA-p ability to evaluate the extent of participation and autonomy of study patients (as a big proportion of people with CVD) in their private and social affairs was "high and very high," showing acceptable face validity. However, some experts (and also some patients) suggested that adding the 'sexual relationship' domain would even increase its coverage. They reported that a written questionnaire could be a suitable opportunity for patients to state (despite its embarrassment) their needs that could improve their quality of life. We concluded that adding this new domain to the IPAp scale, in particular, when applied among CVD patients, might improve its ability to discover more vulnerable domains of patients' life.

Our results also showed a good content validity of IPA-p based on the amounts of CVR $(0.63-1.0)$ and CVI (0.94). According to the Lawshe table, if CVR values are higher than 0.62 and the minimum CVI value is 0.70 , content validity is good $[20,21]$. According to Vazirinejad et al., 60\% of experts believed that the Persian version of IPA was excellent to measure participation and autonomy in
Persian MS (Multiple Sclerosis) patients. Their results suggested the 'religious affairs' domain to be added to IPA-p [12]. Our results confirmed the 'religious affairs' as a helpful domain to be added to the Persian version of the scale. Further to what was done by Vazirinejad et al., content validity was also measured and confirmed based on CVR and CVI in the present study.

Our results showed that the level of participation and autonomy reported by patients via IPA-p was similar to that reported by their relatives, confirming the IPA-p criterion validity to be acceptable.

Materials and factor analysis of IPA-p among positive coronary angiography patients confirmed its construct validity. Although Murano et al. reported a weak correlation between mean scores of different domains of IPA and its total mean score among Japanese diabetic patients [22], our results showed high correlation coefficients ranging between 0.148 and 0.776 , confirming the acceptable construct validity of IPA-p for use among CVD patients. The exploratory factor analysis also showed that the design of IPA-p for applying among CVD patients was acceptable as the model had a good overall fit. In the present study, about $73 \%$ of the variance in the IPA-p overall score was explained by the 9 factors extracted from the model. In Cardol et al., only 4 factors (the original version of IPA) were extracted from the model, being responsible for $68 \%$ of the total IPA scores variance in neurologic patients [9]. The construct validity of the Finnish version of the IPA scale among MS patients was confirmed by Karhula et al. applying confirmatory factor analysis [23].

It should be noted that in our study, the IPA-p scale has 10 domains because the 'religious affairs' domain was added. Although the exploratory factor analysis reduced the number of domains to 9 , the reduced domain was not that of 'religious affairs,' showing its appropriateness. This reduction was due to a few items not completely understood by our respondents. For instance, the third item in the 'paid work' domain that was also 
related to that of 'social relations' was added to this domain by the model. These items could be corrected in the final version of IPA-p for applying among CVD patients.

A tool has good reliability when Cronbach's alpha coefficient is equal to or greater than 0.7 [24]. In the current study, Cronbach's alpha coefficient for the total IPA-p scale was 0.926. The Split-half coefficient and ICC for the scale were 0.825 and 0.363, respectively. Further, the Test-retest coefficient for IPA-p domains ranged between 0.270 and 0.736 , and for the total scale mean score was 0.807 , all confirming the reliability of the IPA-p scale for use among positive coronary angiography patients. We concluded that the IPA-p scale has good reliability for applying among this group of patients. In Cardol et al.'s study, Cronbach's alpha coefficient for IPA ranged from 0.84 to 0.87 among MS patients, indicating good reliability [9]. Ghaziani et al. showed the range of Cronbach's alpha coefficient to be 0.698 to 0.817 for use among Danish patients. Also, the Kappa correlation coefficient was 0.37 to 0.88 , and ICC was reported as 0.701 to 0.818 [25]. Suttiwong et al. showed the Thai version of the IPA to have good reliability with an ICC of 0.76 to 0.96 , and the range of Cronbach's alpha coefficient was 0.86 to 0.96 [26]. Hammar et al. also measured the reliability of IPA among adults aged 70-99 years old, and the range of Test-retest correlation was between 54-100\% [27]. In a different study on neurological patients conducted by Cardol et al., the Cronbach's alpha coefficient of the English version of IPA was 0.81-0.91 [28].

There were some limitations in the present research, including the lack of a representative sample of respondents (both experts and patients) and the unwillingness of some respondents to help with the survey.

\section{Conclusion}

In this study, the face and content validity of the IPA-p scale for applying among positive coronary angiography patients were both confirmed. IPA-p also had acceptable construct and criteria validity, as well as high reliability. Adding some items as the sexual performance domain to the scale might make it more comprehensive for measuring the level of participation and autonomy of Persianlanguage positive angiography patients.

\section{Acknowledgement}

This research was funded by Rafsanjan University of Medical Sciences. We also thank all the experts and patients who helped with this research.
Conflict of interest: None declared.

\section{References}

1. Yavari P. Epidemiological text book of prevalent diseases in Iran. (Non-communicable diseases; Vol 2). Tehran: Gap Publisher; 2018.

2. Myung SK, Ju W, Cho B, Oh SW, Park SM, Koo BK, et al. Efficacy of vitamin and antioxidant supplements in prevention of cardiovascular disease: systematic review and meta- analysis of randomised controlled trials. BMJ 2013; 346:f10.

3. Wang TC, Huang JL, Ho WC, Chiou AF. Effects of a supportive educational nursing care programme on fatigue and quality of life in patients with heart failure: A randomised controlled trial. Eur J Cardiovasc Nurs 2016; 15(2):157-67.

4. Casserley C. Disability, sight impairment, and the law. Br J Ophthalmol 2006; 90(10):1220-2.

5. World Health Organization. Global Data on Visual Impairments 2010. Geneva, Switzerland: World Health Organization; 2012.

6. World Health Organization. International Classification of Impairments, Disabilities, and Handicaps: A manual of classification relating to the consequences of disease. (Published in accordance with resolution WHA29. 35 of the Twenty-ninth World Health Assembly May 1976). Geneva, Switzerland: World Health Organization; 1980.

7. Bray N, Noyes J, Harris N, Edwards RT. Defining health-related quality of life for young wheelchair users: A qualitative health economics study. PLoS 2017; 12(6):e179269.

8. Collin C. Measurement of disability and handicap. In: Greenwood RJ, McMillan TM, Barnes MP, Ward CD, Editor. Handbook of Neurological Rehabilitation. $1^{\text {st }}$ ed. Oxfordshire United Kingdom: Psychology Press; 2003.

9. Cardol M, de Haan RJ, van den Bos GA, de Jong BA, de Groot IJ. The development of a handicap assessment questionnaire: the Impact on Participation and Autonomy (IPA). Clin Rehabil 1999; 13(5):411-9.

10. Fletcher RH, Fletcher SW, Fletcher GS. Clinical Epidemiology: The Essentials. [Dadgarmoghadam $M$, Janghorbani $M$, Nirumand $\mathrm{SH}$, KHademrezaeian $\mathrm{M}$, Sadeghihassanabadi A, Trans]. 1st ed. Tehran: Gap Nashr; 2015. p.56-60.

11. Dixon JK. Factor Analysis. In: Kellar SP, Kelvin E, Editor. Munro's Statstical Methods for Health Care Research. $6^{\text {th }}$ ed. Philadeiphia, United States: Lippincot Williams\& Wilkins; 2012.

12. Vazirinejad R, Joorian J, Taghavi MM, Lilley JM, Sayadi Anari AR. The Persian Version of a Participation Scale: Is It Valid and Reliable Enough for Use among Iranian Patients with Multiple Sclerosis. J Clin Neurol 2015; 11(2):157-63. 
13. Lawshe CH. A Quantitative Approach to Content Validity. Perssonel Psychology 1975; 28(4):563-75.

14. Saraie H. An Introduction on Sampling in Research. 10th ed. Tehran: Samt Publish; 2017.

15. Ghamarani A, Siadatian SH, Pishdad R. An Investigation of Validity and Reliability of Entrapment Scale in the Students of Isfahan University of Medical Sciences, Iran. Qom Univ Med Sci J 2014; 7(6):54-61.

16. Brace N, Snelgar RS, Kemp R. SPSS for Psychologists. 5th ed. London, United Kingdom: Palgrave Macmillan; 2012.

17. Mohammadbeigi A, Mohammadsalehi N, Aligol M. Validity and Reliability of the Instruments and Types of Measurements in Health Applied Researches. J Rafsanjan Univ Med Sci 2015; 13(12):1153-70.

18. Sharif Nia $\mathrm{H}$, Ebadi $\mathrm{A}$, Lehto $\mathrm{RH}$, Mousavi $\mathrm{B}$, Peyrovi $\mathrm{H}$, Chan $\mathrm{YH}$. Reliability and validity of the persian version of templer death anxiety scale-extended in veterans of Iran-Iraq warfare. Iran J Psychiatry Behav Sci 2014; 8(4):29-37.

19. Tabachnick BG, Fidle LS. Using Multivariate Statistics. 7th ed. Boston, Massachusetts, United States: Pearson Education; 2019.

20. Hall DE, Lynn JM, Altieri J, Segers VD, Conti D. Inter- intrajudge reliability of the stuttering severity instrument. J Fluency Disord 1987; 12(3):167-73.

21. Delshad $M H$, Hidarnia $A$, Niknam $S$. Psychometric measure continuous variables preventive behaviors of hepatitis B virus infection in health care workers. J Mazandaran
Univ Med Sci 2014; 23(109):71-82.

22. Murano I, Asakawa Y, Mizukami M, Takihara J. Examination of the Validity of the Japanese Version of the IPA Questionnaire for the Evaluation of the Physical Activity of Diabetes Patients. Rigaku Roho Sci 2013; 28:101-4.

23. Karhula $M E$, Salminen $A L$, Hämäläinen $P I$, Ruutiainen J, Era P, Tolvanen A. Psychometric evaluation of the Finnish version of the impact on participation and autonomy questionnaire in persons with multiple sclerosis. Scand J Occup Ther 2017; 24(6):410-20.

24. Plitcha Kellar S, Kelvin E. Munros Statistical Methods for Health Care Research. 6th ed. Philadelphia, United States: Lippincott Wiliams and Wikins; 2012. p.14-34.

25. Ghaziani E, Krogh AG, Lund H. Developing a Danish version of the "Impact on Participation and Autonomy Questionnaire". Scand J Occup Ther 2013; 20(3):190-200.

26. Suttiwong J, Vongsirinavarat $M$, Vachalathiti R, Chaiyawat $P$. Impact on participation and autonomy questionnaire: psychometric properties of the thai version. J Phys Ther Sci 2013; 25(7):769-74.

27. Hammar IO, Ekelund C, Wilhelmson K, Eklund K. Impact on Participation and Autonomy: Test of Validity and Reliability for Older Persons. Health Psychol Res 2014; 2(3):1825.

28. Cardol M, de Haan RJ, de Jong $B A$, van den Bos GA, de Groot IJ. Psychometric properties of the Impact on Participation and Autonomy Questionnaire. Arch Phys Med Rehabil 2001; 82(2):210-6. 